\title{
Wave Trains Associated with a Cascade of Bifurcations of Space-Time Caustics over Elongated Underwater Banks
}

\author{
S. Yu. Dobrokhotov ${ }^{1,2} *$, D. A. Lozhnikov ${ }^{1,2}$, V. E. Nazaikinskii ${ }^{1,2}$ \\ ${ }^{1}$ A. Ishlinsky Institute for Problems in Mechanics of the Russian Academy of Sciences \\ ${ }^{2}$ Moscow Institute of Physics and Technology
}

\begin{abstract}
We study the behavior of linear nonstationary shallow water waves generated by an instantaneous localized source as they propagate over and become trapped by elongated underwater banks or ridges. To find the solutions of the corresponding equations, we use an earlier-developed asymptotic approach based on a generalization of Maslov's canonical operator, which provides a relatively simple and efficient analytic-numerical algorithm for the wave field computation. An analysis of simple examples (where the bank and source shapes are given by certain elementary functions) shows that the appearance and dynamics of trapped wave trains is closely related to a cascade of bifurcations of space-time caustics, the bifurcation parameter being the bank length-to-width ratio.
\end{abstract}

Keywords and phrases: linearized shallow water equations, asymptotics, wave front singularity evolution, trapped nonstationary wave, underwater ridge, underwater bank

Mathematics Subject Classification: 37C23, 76B15, 74J05, 35A18

\section{Introduction}

Wave focusing, caustics, wavefront singularities, etc. normally occur in an inhomogeneous medium or in a medium confined in a domain with variable boundary. The wave field amplitude increases in the vicinity of such phenomena, which makes them interesting from the physical point of view. A caustic is often treated as an individual object; on the other hand, it is of interest how the presence of a set of caustics influences the global wave field. This influence was considered by Berry and his collaborators ([3]; see also [4]) in the context of optics. Here we discuss close effects in water wave theory and study long waves generated by instantaneous localized sources and propagating over elongated underwater banks and ridges. Namely, we are interested in the asymptotics of the solution of the following Cauchy problem

\footnotetext{
${ }^{*}$ Corresponding author. E-mail: dobr@ipmnet.ru
} 
for the linearized shallow water equations in dimensionless variables on the two-dimensional plane $\mathbb{R}_{x}^{2}$ :

$$
\begin{aligned}
& \frac{\partial \eta}{\partial t}+\operatorname{div}\left(C^{2}(x) u\right)=0, \quad \frac{\partial u}{\partial t}+\nabla \eta=0, \quad \text { where } C(x)=\sqrt{g D(x)}, \quad x=\left(x_{1}, x_{2}\right) \in \mathbb{R}^{2}, \\
& \left.\eta\right|_{t=0}=\eta^{0}\left(\frac{x-x^{0}}{\mu}\right),\left.\quad u\right|_{t=0}=0 .
\end{aligned}
$$

Here $\eta=\eta(x, t)$ is the free surface elevation, the 2-vector $u=u(x, t)$ is the horizontal velocity, $D(x)$ is the (smooth) basin depth function, $g$ is the acceleration due to gravity, the initial source function $\eta^{0}(z)$, $z=\left(z_{1}, z_{2}\right)$, is smooth and decays at infinity more rapidly than $1 /|z|^{1+\varkappa}, \varkappa>0$, and the 2-vector $x^{0}$ specifies the source position. This problem is equivalent to the Cauchy problem

$$
\eta_{t t}-\nabla C^{2}(x) \nabla \eta=0,\left.\quad \eta\right|_{t=0}=\eta^{0}\left(\frac{x-x^{0}}{\mu}\right),\left.\quad \eta_{t}\right|_{t=0}=0
$$

for the two-dimensional wave equation with the same velocity $C(x)=\sqrt{g D(x)}$.

Our situation is different from that discussed in [3] in that we deal with waves localized in the vicinity of closed curves (fronts) rather than rapidly oscillating everywhere on $\mathbb{R}_{x}^{2}$. Here, as well as in [3], one can observe a cascade of caustics under certain conditions on the velocity $C(x)$, but these are spacetime caustics in our case. The wave fronts are the curves (in general, with turning points and points of self-intersection) near which the waves are localized; they are constant phase lines, just as in the case of oscillating waves. At each instant of time, the localized waves can be viewed as solitary waves in the direction normal to the wave front. We claim that such cascades of bifurcations of caustics result in wave trains and underlie the phenomenon of trapped waves propagating over elongated underwater banks and ridges.

Let us say a few words about this phenomenon. It is well known that underwater ridges and banks can produce trapped waves. Usually they are treated as stationary or quasi-stationary states of the 3D water wave problem or of the $2 \mathrm{D}$ Beltrami-Laplace operator $-\nabla C^{2}(x) \nabla$ if the long wave approximation is used. Trapped waves are used to explain many effects in ocean physics, in particular, the fact that tsunami waves can travel long distances along ocean ridges without energy scattering.

There is a vast literature on the topic, and we mention only a few papers and monographs $[2,18,19$, $21,24,25,28]$, where one can find further references. Stationary problems for trapped waves have usually been considered for the case in which the depth function $D(x)$ depends only on the variable $x_{1}$ or the polar radius $r=\sqrt{x_{1}^{2}+x_{2}^{2}}$. To construct analytical formulas in a more general situation, one should use asymptotic methods (e.g., see $[5,7,14,20,23]$ ). Wave propagation over underwater ridges in the nonstationary setting is not well studied. Our goal is to present simple asymptotic formulas and carry out some computations for a simple model example so as to demonstrate the relationship between trapped nonstationary waves and cascades of caustics produced by elongated banks. Numerical analysis of the corresponding Hamiltonian system shows that the number of caustics generated by the bank depends on the bank length-to-width ratio $q$ (as well as on the depth over the bank and the bank height), so that there is a cascade of bifurcations of caustics in the system. For fundamentals of bifurcation theory, we refer the reader to $[1,16,27]$.

\section{Waves generated by a localized source over a nonuniform bottom}

Our analysis is based on the efficient asymptotic formulas obtained for the solutions of the Cauchy problem (1.1), (1.2) in the papers [6,9-13]. According to these papers, the solution of problem (1.1), (1.2) splits into two parts, one corresponding to the propagation of a solitary vortex and the other corresponding to wave propagation. The description of each of these parts can be given in terms of the trajectories of a Hamiltonian system. Here we omit the vortical part and only deal with the wave part and the equivalent problem (1.3). In the four-dimensional phase space $\mathbb{R}_{x, p}^{4}$ with coordinates $x=\left(x_{1}, x_{2}\right)$ 
and $p=\left(p_{1}, p_{2}\right)$, consider the following Cauchy problem ${ }^{1}$ for the Hamiltonian system with Hamiltonian $H(x, p)=|p| C(x)$ in $\mathbb{R}_{p, x}^{4}$ :

$$
\dot{x}=H_{p} \equiv \frac{p}{|p|} C(x), \quad \dot{p}=-H_{x} \equiv-|p| \nabla C(x),\left.\quad p\right|_{t=0}=\mathbf{n}(\psi),\left.\quad x\right|_{t=0}=x^{0}, \quad \psi \in[0,2 \pi),
$$

where $\mathbf{n}(\psi)=(\cos \psi, \sin \psi)$. Let $x=X(\psi, t), p=P(\psi, t)$ be the solution of problem (2.1). For further reference, note the following easy-to-verify properties of these functions:

$$
\begin{gathered}
\langle P(\psi, t), \dot{X}(\psi, t)\rangle=C\left(x^{0}\right), \quad\left\langle P(\psi, t), X_{\psi}(\psi, t)\right\rangle=\left\langle\dot{X}(\psi, t), X_{\psi}(\psi, t)\right\rangle=0, \\
|P(\psi, t)|=\frac{C\left(x^{0}\right)}{C(X(\psi, t))}, \quad|\dot{X}(\psi, t)|=C(X(\psi, t)), \quad \operatorname{rank} \frac{\partial(X(\psi, t), P(\psi, t))}{\partial(\psi, t)}=2,
\end{gathered}
$$

where the angle brackets $\langle\cdot, \cdot\rangle$ stand for the inner product on $\mathbb{R}^{2}$ and $|\xi|=\langle\xi, \xi\rangle^{1 / 2}$. For each $\psi$, the functions $(X(\psi, t), P(\psi, t))$ define a curve known as a bicharacteristic (characteristic in the phase space) of problem (1.3). The closed smooth curve $\Gamma_{t}$ formed by the points of these bicharacteristics for given $t$ as $\psi$ varies in $[0,2 \pi)$ is called the (time $t$ ) wave front in the phase space. The projection

$$
\gamma_{t}=\{x=X(\psi, t): \psi \in[0,2 \pi)\}
$$

of $\Gamma_{t}$ onto the two-dimensional configuration space $\mathbb{R}_{x}^{2}$ is called the wave front on the plane (in the configuration space), or simply the wave front. The solution of problem (1.3) is asymptotically localized in a neighborhood of $\gamma_{t}$ as $\mu \rightarrow 0 .^{2}$

Unlike $\Gamma_{t}$, the wave front $\gamma_{t}$ is not necessarily smooth. For $t=0$, it degenerates into the point $x^{0}$ (in complete agreement with the fact that the solution is localized near the source position $x^{0}$ at the initial time). Next, for small $t>0$, the wave front is a smooth curve (resembling a distorted circle), but for larger $t$ it may have turning points and points of self-intersection. The turning points on $\gamma_{t}$ (and the corresponding focal points on $\Gamma_{t}$ ) are the points where $X_{\psi}=0$. The points where $X_{\psi} \neq 0$ are said to be regular. The locus of turning points in the three-dimensional space-time $\mathbb{R}_{x, t}^{3}$ is called the space-time caustic and in general position looks like a cusp. If the underwater bank (or ridge) is sufficiently long, then, for larger $t$, new singular points emerge on the front; they form new space-time caustics, and thus a cascade of such caustics may appear depending on the bank parameters. The solution amplitude is known to increase near the caustics, and so this geometrical-optics phenomenon of a cascade of caustics in the characteristics (2.1) manifests itself in the solution of problem (1.3) as a trapped wave train propagating over the ridge.

After this qualitative description of the solution of problem (1.3), let us write out the asymptotic formulas for the free surface elevation $\eta$ as $\mu \rightarrow 0$. They depend on whether we deal with a neighborhood of a regular or a turning point of the wave front, and we consider the two cases separately.

\subsection{Wave field near the regular points}

One can specify a point $x$ in a neighborhood of a regular point $x_{*}=X\left(\psi_{*}, t_{*}\right) \in \gamma_{t_{*}}$ by the coordinates $(\psi(x, t), y(x, t))$, where $\psi(x, t)$ is determined from the condition

$$
\left\langle x-X(\psi, t), X_{\psi}(\psi, t)\right\rangle=0
$$

of orthogonality of the vector $x-X(\psi, t)$ to the vector $X_{\psi}$ tangent to $\gamma_{t}$ at the point $X(\psi, t)$ and $y(x, t)$ is the signed distance between $x$ and $X(\psi(x, t), t)$. Next, we need the Morse index $m=m(\psi, t)$ of the

\footnotetext{
${ }^{1}$ In (2.1) and similar formulas below, $p$ and $x$ are treated as column vectors.

${ }^{2}$ Note, however, that the maximum of $|\eta|$ on a line normal to $\gamma_{t}$ is not necessarily attained exactly on $\gamma_{t}$; the points of maximum of $|\eta|$ are just very close to $\gamma_{t}$.
} 
trajectory $\{X(\psi, \tau), \tau \in(0, t]\}$ defined as the number of turning points on this trajectory, or, which is the same, the number of sign changes of the Jacobian

$$
J(\psi, \tau)=\operatorname{det}\left(\dot{X}(\psi, \tau), X_{\psi}(\psi, \tau)\right)
$$

where $\left(\dot{X}, X_{\psi}\right)$ is the $2 \times 2$ matrix with columns $\dot{X}$ and $X_{\psi}$, on the interval $(0, t]$. (In view of $(2.2)$, one has

$$
|J(\psi, \tau)|=|\dot{X}(\psi, \tau)|\left|X_{\psi}(\psi, \tau)\right|=C(X(\psi, \tau))\left|X_{\psi}(\psi, \tau)\right|,
$$

so that the Jacobian $J(\psi, t)$ vanishes exactly at the same points as $X_{\psi}(\psi, t)$.) Clearly, $m(\psi, t)$ is constant in a neighborhood of any regular point. Finally, we introduce the phase function

$$
S(x, t)=\langle P(\psi(x, t), t), x-X(\psi(x, t), t)\rangle=y(x, t) \sqrt{\frac{D\left(x^{0}\right)}{D(X(\psi(x, t), t))}}
$$

and the shape function

$$
F(s, \psi)=\frac{e^{-i \pi / 4}}{\sqrt{2 \pi}} \int_{0}^{\infty} \widetilde{\eta}^{0}(\rho \mathbf{n}(\psi)) \sqrt{\rho} e^{i s \rho} d \rho
$$

where

$$
\widetilde{\eta}^{0}(k)=\frac{1}{2 \pi} \int_{\mathbb{R}^{2}} \eta^{0}(z) e^{i\langle k, z\rangle} d z, \quad k=\left(k_{1}, k_{2}\right), \quad z=\left(z_{1}, z_{2}\right),
$$

is the Fourier transform of $\eta^{0}(z)$. The asymptotics of the solution of problem (1.3) in a neighborhood of the point $\left(x_{*}, t_{*}\right)$ has the form [12]

$$
\eta(x, t)=\left.\sqrt{\frac{\mu C\left(x^{0}\right)}{\left|X_{\psi}(\psi, t)\right| C(X(\psi, t), t)}} \operatorname{Re}\left[e^{-i \pi m\left(\psi_{*}, t_{*}\right) / 2} F\left(\frac{S(x, t)}{\mu}, \psi\right)\right]\right|_{\psi=\psi(x, t)}+o\left(\mu^{1 / 2}\right) .
$$

Remark 2.1. If $x_{*}$ is a point of self-intersection of $\gamma_{t_{*}}$, then for the asymptotics in a neighborhood of $x_{*}$ one should take the sum of all contributions (2.6) of the branches of $\gamma_{t_{*}}$ that meet at $x_{*}$; if, for some of these branches, $x_{*}$ is a turning point, then the corresponding contributions are given by formula $(2.10)$ below rather than by $(2.6)$.

\subsection{Wave field near the caustics}

Let $\left(x_{*}, p_{*}\right)=\left(X\left(\psi_{*}, t_{*}\right), P\left(\psi_{*}, t_{*}\right)\right)$ be a focal point on $\Gamma_{t_{*}}$. The Jacobian

$$
\widetilde{J}(\psi, t)=\operatorname{det}\left(P(\psi, t), P_{\psi}(\psi, t)\right)
$$

(where $\left(P(\psi, t), P_{\psi}(\psi, t)\right)$ is the $2 \times 2$ matrix with columns $P(\psi, t)$ and $\left.P_{\psi}(\psi, t)\right)$ is nonzero at the point $\left(\psi_{*}, t_{*}\right)$. Indeed,

$$
\left.\left.|\widetilde{J}(\psi, t)|=|P(\psi, t)| \mid P_{\psi}(\psi, t)\right)|| \sin \alpha(\psi, t)\left|=\frac{C\left(x^{0}\right)}{C(X(\psi, t))}\right| P_{\psi}(\psi, t)\right)|| \sin \alpha(\psi, t) \mid,
$$

where $\alpha(\psi, t)$ is the angle between the vectors $P(\psi, t)$ and $\left.P_{\psi}(\psi, t)\right)$. Since $X_{\psi}\left(\psi_{*}, t_{*}\right)=0$, it follows from the rank condition in $(2.2)$ that $P_{\psi}\left(\psi_{*}, t_{*}\right) \neq 0$. Finally, the vectors $P_{\psi}\left(\psi_{*}, t_{*}\right)$ and $P\left(\psi_{*}, t_{*}\right)$ are orthogonal, which readily follows by differentiating the relation $|P(\psi, t)| C(X(\psi, t))=$ const, and so $\sin \alpha\left(\psi_{*}, t_{*}\right)= \pm 1$. Let $t(\psi, x)$ be the local implicit function determined by the equation

$$
\langle P(\psi, t), x-X(\psi, t)\rangle=0, \quad t\left(\psi_{*}, x_{*}\right)=t_{*},
$$


and let $\mathbf{e}(\psi, t)$ be a cutoff function supported in a sufficiently small neighborhood of $\left(\psi_{*}, t_{*}\right)$, so that $\widetilde{J}(\psi, t) \neq 0$ on the support of $\mathbf{e}(\psi, t)$. The asymptotics of the solution of problem (1.3) in a neighborhood of the point $x_{*}$ has the form (see, however, Remark 2.1 above)

$$
\begin{aligned}
& \eta(x, t)=\operatorname{Re}\left[\frac{1}{2 \pi} e^{-i \pi m\left(\psi_{*}, t_{*}\right) / 2} \sqrt{C\left(x^{0}\right)}\right. \\
&\left.\times \int_{0}^{\infty}\left(\int_{-\infty}^{\infty} e^{i \rho C\left(x^{0}\right)(t(\psi, x)-t) / \mu} \sqrt{|\widetilde{J}(\psi, t(\psi, x))|} \mathbf{e}(\psi, t(\psi, x)) \widetilde{\eta}^{0}(\rho \mathbf{n}(\psi)) d \psi\right) \rho d \rho\right]+o\left(\mu^{1 / 2}\right) .
\end{aligned}
$$

This representation is new and replaces another, more complicated formula based on the standard version of Maslov's canonical operator (and involving mixed coordinate-momentum representations), which was used in $[13,22]$. We omit the derivation of formula $(2.10)$; it is based on a new representation of the canonical operator, which will be discussed in detail in a forthcoming paper by the authors. Here we only explain how to recover (2.6) from (2.10) near a regular point of the wave front close to $\left(x_{*}, t_{*}\right)$. Thus, consider such a regular point $\left(x_{\circ}, t_{\circ}\right), x_{\circ}=X\left(\psi_{\circ}, t_{\circ}\right), t\left(\psi_{\circ}, x_{\circ}\right)=t_{\circ}$, where $t_{\circ}$ is close to $t_{*}$ and $\psi_{\circ}$ is close to $\psi_{*}$.

Proposition 2.2. Let $(x, t)$ vary in a sufficiently small neighborhood of $\left(x_{\circ}, t_{\circ}\right)$. Then the expressions (2.6) and (2.10) coincide modulo o $\left(\mu^{1 / 2}\right)$.

Proof. Let us compute the asymptotics as $\mu \rightarrow \infty$ of the integral over $\psi$ in (2.10) by the stationary phase method [17, Chap. 3]. The stationary point equation reads

$$
\frac{\partial t}{\partial \psi}(\psi, x) \equiv \frac{\left\langle P_{\psi}(\psi, t(\psi, x)), x-X(\psi, t(\psi, x))\right\rangle}{1+\langle\dot{P}(\psi, t(\psi, x)), X(\psi, t(\psi, x))-x\rangle}=0 .
$$

(To compute the derivative $\partial t(\psi, x) / \partial \psi$, we have taken the differential of the left-hand side of $(2.9)$ and used relations (2.2).) Together with (2.9), this gives

$$
\langle P(\psi, t(\psi, x)), x-X(\psi, t(\psi, x))\rangle=0, \quad\left\langle P_{\psi}(\psi, t(\psi, x)), x-X(\psi, t(\psi, x))\right\rangle=0,
$$

and since the Jacobian (2.7) is nonzero, we see that

$$
x-X(\psi, t(\psi, x))=0
$$

at the stationary points. For $x=x_{\circ}$, the stationary point equation has the solution $\psi=\psi_{\circ}$. By differentiating the left-hand side of (2.11), we find that

$$
\frac{\partial^{2} t}{\partial \psi^{2}}(\psi, x)=-\left\langle P_{\psi}(\psi, t(\psi, x)), X_{\psi}(\psi, t(\psi, x))\right\rangle
$$

at the stationary points. Since the vectors $P(\psi, t)$ and $X_{\psi}(\psi, t)$ are orthogonal by $(2.2)$, we have

$$
\left|\frac{\partial^{2} t}{\partial \psi^{2}}(\psi, x)\right|=\left|\left\langle P_{\psi}(\psi, t(\psi, x)), X_{\psi}(\psi, t(\psi, x))\right\rangle\right|=\left|P_{\psi}(\psi, t(\psi, x))\right|\left|X_{\psi}(\psi, t(\psi, x))\right||\sin \alpha(\psi, t(\psi, x))|,
$$

where $\alpha(\psi, t)$ is the angle between the vectors $P(\psi, t)$ and $\left.P_{\psi}(\psi, t)\right)$. By combining this with $(2.8)$, we obtain

$$
\left|\frac{\partial^{2} t}{\partial \psi^{2}}(\psi, x)\right|=\frac{C(X(\psi, t(\psi, x)))}{C\left(x^{0}\right)}\left|X_{\psi}(\psi, t(\psi, x))\right||\widetilde{J}(\psi, t(\psi, x))| .
$$

This expression is nonzero at the point $\left(\psi_{\circ}, x_{\circ}\right)$, because $X_{\psi}\left(\psi_{\circ}, t_{\circ}\right) \neq 0$ (the point $\left(x_{\circ}, t_{\circ}\right)$ is nonsingular) and $\left.\widetilde{J}\left(\psi_{\circ}, t_{\circ}\right)\right) \neq 0$ (the point $\left(x_{\circ}, t_{\circ}\right)$ is close to the singular point $\left.\left(x_{*}, t_{*}\right)\right)$. By the implicit function theorem, there exists a unique nondegenerate stationary point $\psi=\Psi(x)$ for $x$ close to $x_{\circ}, \Psi\left(x_{\circ}\right)=\psi_{\circ}$, 
and we can apply the the stationary phase method to the inner integral in (2.10), thus obtaining, in view of $(2.13)$,

$$
\begin{aligned}
\eta(x, t)=\operatorname{Re}\left[e^{-i \pi\left(m\left(\psi_{*}, t_{*}\right)-\theta\left(-t_{\psi \psi}^{\prime \prime}(\Psi(x), T(x))\right)\right) / 2} \sqrt{\frac{\mu C\left(x^{0}\right)}{\left|X_{\psi}(\Psi(x), T(x))\right| C(x)}}\right. \\
\left.\quad \times \frac{e^{-i \pi / 4}}{\sqrt{2 \pi}} \int_{0}^{\infty} e^{i \rho C\left(x^{0}\right)(T(x)-t) / \mu} \widetilde{\eta}^{0}(\rho \mathbf{n}(\Psi(x))) \sqrt{\rho} d \rho\right]+o\left(\mu^{1 / 2}\right),
\end{aligned}
$$

where $\theta(v)=(1+\operatorname{sign} v) / 2$ is the Heaviside function and $T(x)=t(\Psi(x), x)$. Note that the pair $(\Psi(x), T(x))$ is the solution of the system $x=X(\Psi, T)$ in a neighborhood of $\left(\psi_{\circ}, t_{\circ}\right)$. Let us simplify the expression (2.14). The integral on the second line gives the shape function (2.5), and it can be shown that

$$
m\left(\psi_{*}, t_{*}\right)-\theta\left(-t_{\psi \psi}^{\prime \prime}(\Psi(x), T(x))\right)=m\left(\psi_{\circ}, t_{\circ}\right)
$$

thus, we obtain

$$
\begin{aligned}
\eta(x, t)=\operatorname{Re}\left[e^{-i \pi m\left(\psi_{\circ}, t_{\circ}\right) / 2} \sqrt{\frac{\mu C\left(x^{0}\right)}{\left|X_{\psi}(\Psi(x), T(x))\right| C(x)}}\right. \\
\left.\left.\times F\left(\frac{C\left(x^{0}\right)(T(x)-t)}{\mu}, \Psi(x)\right)\right)\right]+o\left(\mu^{1 / 2}\right) .
\end{aligned}
$$

A straightforward computation shows that

$$
\begin{gathered}
C\left(x^{0}\right)(T(x)-t)=S(x, t)+O\left(S(x, t)^{2}\right), \\
T(x)-t=O(S(x, t)), \quad \Psi(x)-\psi(x, t)=O\left(S(x, t)^{2}\right)
\end{gathered}
$$

in a neighborhood of $\left(\psi_{\circ}, t_{\circ}\right)$, where $S(x, t)$ is the phase function $(2.4)$. Indeed,

$$
\begin{aligned}
S(x, t)= & \langle P(\psi(x, t), t), x-X(\psi(x, t), t)\rangle=\langle P(\psi(x, t), t), X(\Psi(x), T(x))-X(\psi(x, t), t)\rangle \\
= & \left\langle P(\psi(x, t), t), X_{\psi}(\psi(x, t), t)\right\rangle(\Psi(x)-\psi(x, t)) \\
& \quad+\langle P(\psi(x, t), t), \dot{X}(\psi(x, t), t)\rangle(T(x)-t)+O\left((\Psi(x)-\psi(x, t))^{2}+(T(x)-t)^{2}\right) \\
= & C\left(x^{0}\right)(T(x)-t)+O\left((\Psi(x)-\psi(x, t))^{2}+(T(x)-t)^{2}\right)
\end{aligned}
$$

by (2.2). Next, from (2.3), with regard to (2.2), we have

$$
\begin{aligned}
0 & =\left\langle X(\Psi(x), T(x))-X(\psi(x, t), t), X_{\psi}(\psi(x, t), t)\right\rangle \\
& =\left|X_{\psi}(\psi(x, t), t)\right|^{2}(\Psi(x)-\psi(x, t))+O\left((\Psi(x)-\psi(x, t))^{2}+(T(x)-t)^{2}\right)
\end{aligned}
$$

and hence

$$
\Psi(x)-\psi(x, t)=O\left((T(x)-t)^{2}\right)
$$

in a neighborhood of $\left(\psi_{\circ}, t_{\circ}\right)$. By combining (2.17) with (2.18), we readily arrive at $(2.16)$.

Since the function $F(s, \psi)$ decays as $s \rightarrow \infty$, it follows from (2.16) that the expression (2.15) coincides with (2.6) modulo $o\left(\mu^{1 / 2}\right)$. The proof of the proposition is complete. (We have omitted some technical details, which can be found for a similar situation in [13].)

\section{Bifurcation of space-time caustics and cascades of caustics}

Now we proceed to a simple example illustrating our general considerations. Consider the underwater bank determined by the basin depth function

$$
D\left(x_{1}, x_{2}\right)=1-\frac{a_{0}}{1+\left(\left(x_{1}-a_{1}\right) / b_{1}\right)^{2}+\left(\left(x_{2}-a_{2}\right) / b_{2}\right)^{2}},
$$


where $a_{0}, a_{1}, a_{2}, b_{1}$, and $b_{2}$ are positive parameters. The source is placed at the origin, $x^{0}=(0,0)$. Furthermore, we use the parameter values $a_{0}=0.5, a_{1}=0, a_{2}=350$, and $b_{1}=40$ in all the computations and vary only the parameter $b_{2}$, which plays the role of a bifurcation parameter in our analysis.

Figures 1-4 show parts of the wave front at the successive instants of time $t_{1}=400, t_{2}=500$, $t_{3}=1000, t_{4}=1500$, and $t_{5}=2000$ as the wave generated by a localized source at $t=0$ passes over the elongated underwater banks given by the expression (3.1). The wave fronts were computed in the sector $\psi \in[\pi / 4,3 \pi / 4]$. The bank length parameter is taken to be $b_{2}=95$ for Fig. $1, b_{2}=120$ for Fig. 2, $b_{2}=180$ for Fig. 3, and $b_{2}=280$ for Fig. 4. All figures also display the bottom relief (the level lines of the function (3.1)), and the source is marked as a bold dot at the origin. The dashed lines show the space-time caustics. We see that the larger $b_{2}$, the more space-time caustics are there in the problem. In other words, the computations suggest that there exists a cascade of bifurcations of caustics with bifurcation parameter $b_{2}$ (or, equivalently, the length-to-width ratio $q=b_{2} / b_{1}$ ).

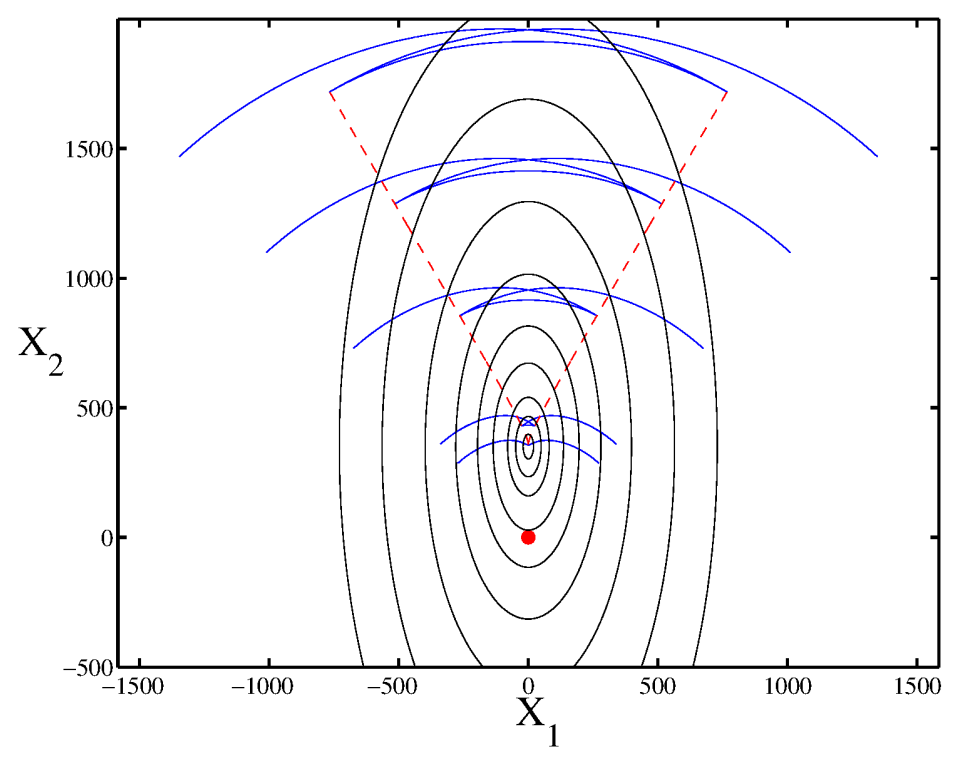

FiguRe 1.

\section{Trapped wave trains associated with cascades of caustics}

Let us show how the wave field is computed in our example according to the asymptotic formulas (2.6) and (2.10). To simplify the formulas, we use a special choice of $\eta^{0}(z)$; namely,

$$
\eta^{0}(z)=\frac{A}{\left(1+\left(z_{1} / B_{1}\right)^{2}+\left(z_{2} / B_{2}\right)^{2}\right)^{3 / 2}},
$$

where $B_{1}, B_{2}$, and $A$ are positive parameters. This source, which generalizes the one used in $[15,25,29]$, has very useful and apparently unique properties (see $[6,8,11-13,26])$ and provides an opportunity to express the asymptotic solution formulas in terms of elementary functions. The last property is related to the special form of the Fourier transform of this function,

$$
\widetilde{\eta}^{0}(\rho \mathbf{n}(\psi))=A \cdot e^{-\rho \beta(\psi)}, \quad \beta(\psi)=\sqrt{B_{1}^{2} \cos ^{2} \psi+B_{2}^{2} \sin ^{2} \psi} .
$$




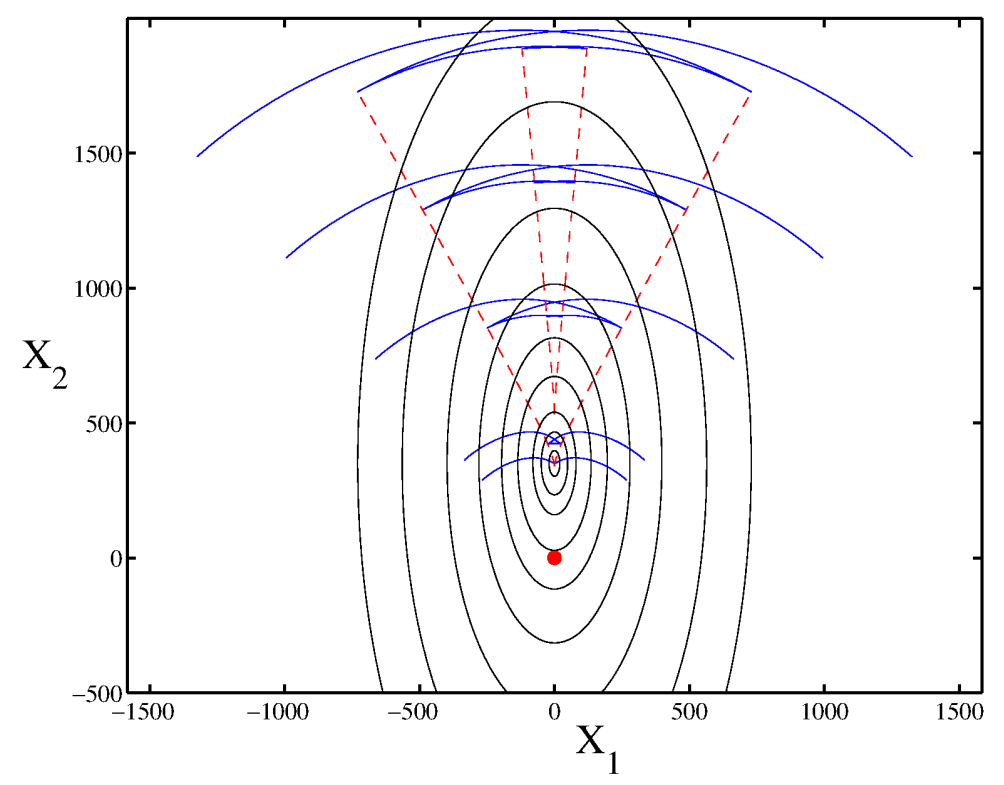

FIGURE 2.

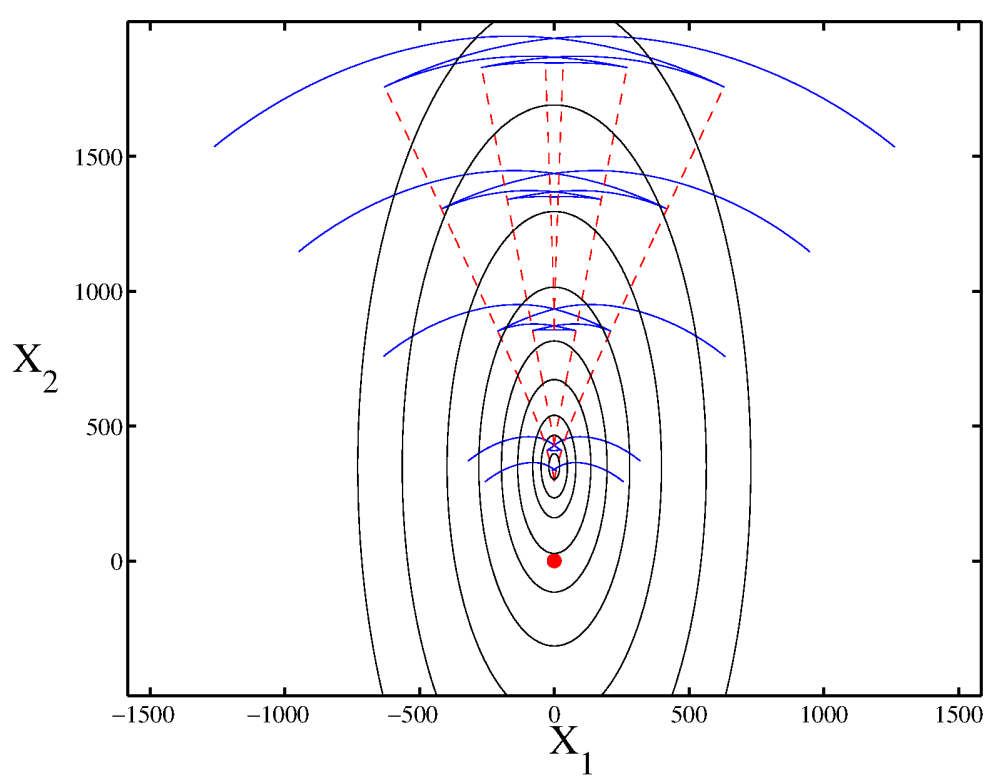

Figure 3.

Owing to this simple form, one can express the integral (2.5) via elementary algebraic functions,

$$
F(s, \psi)=\frac{A e^{-i \frac{\pi}{4}}}{2 \sqrt{2}\left(\sqrt{B_{1}^{2} \cos ^{2} \psi+B_{2}^{2} \sin ^{2} \psi}-i s\right)^{3 / 2}} .
$$




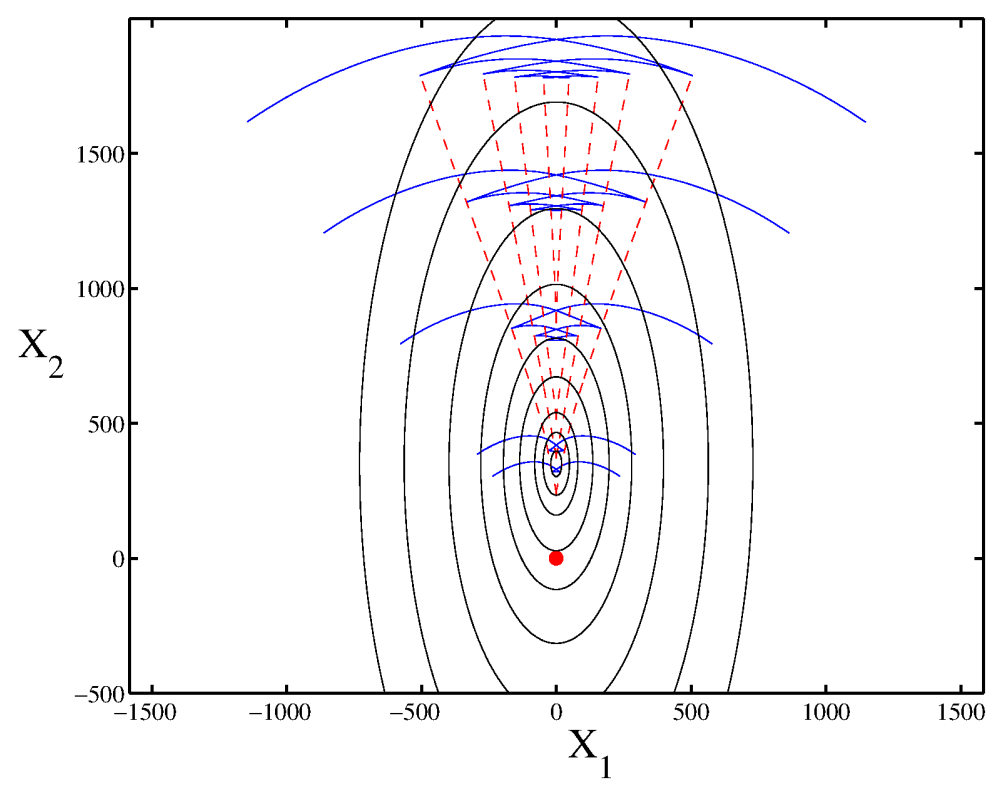

FIGURE 4.

where

$$
\arg \left(\sqrt{B_{1}^{2} \cos ^{2} \psi+B_{2}^{2} \sin ^{2} \psi}-i s\right) \in\left(-\frac{\pi}{2}, \frac{\pi}{2}\right) .
$$

The function (2.10) can be expressed in terms of algebraic functions in this case as well. However, the corresponding expression is somewhat more awkward than (4.2), and we omit it here; it can be found in $[13,22]$. Figure 5 shows the initial free surface elevation (4.1).

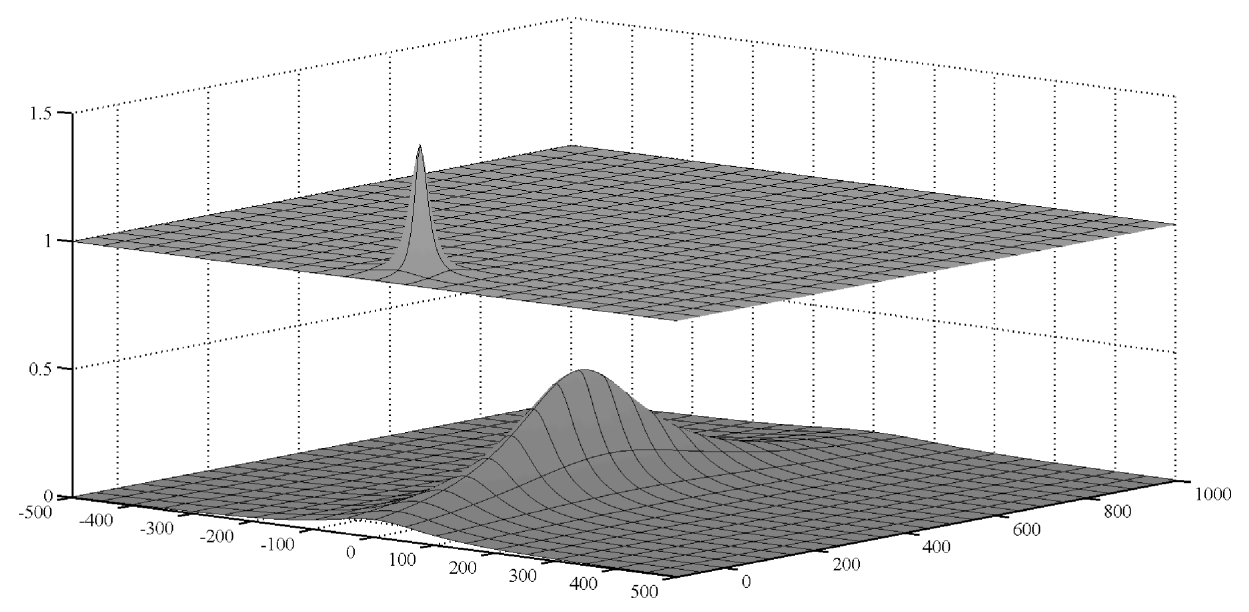

FiguRE 5. The initial free water surface elevation over an elliptical underwater bank, shown schematically. (The actual source size is substantially smaller.) 
Now let us describe the computations themselves. First, one should compute the Jacobians $J=$ $\operatorname{det}\left(\dot{X}, X_{\psi}\right)$ and $\widetilde{J}=\operatorname{det}\left(P, P_{\psi}\right)$ to find the domains where the representations (2.6) and (2.10) can be used. (These representations hold where the respective Jacobians are nonzero.) By way of example, Figure 6 shows the graphs of the Jacobians $J$ (dashed line) and $\widetilde{J}$ (solid line) at time $t=2000$ for the bifurcation parameter value $b_{2}=280$ (which corresponds to Fig. 4). Solid vertical lines correspond to focal points. After that, it remains to use formulas (2.6) and (2.10) in the corresponding domains. A

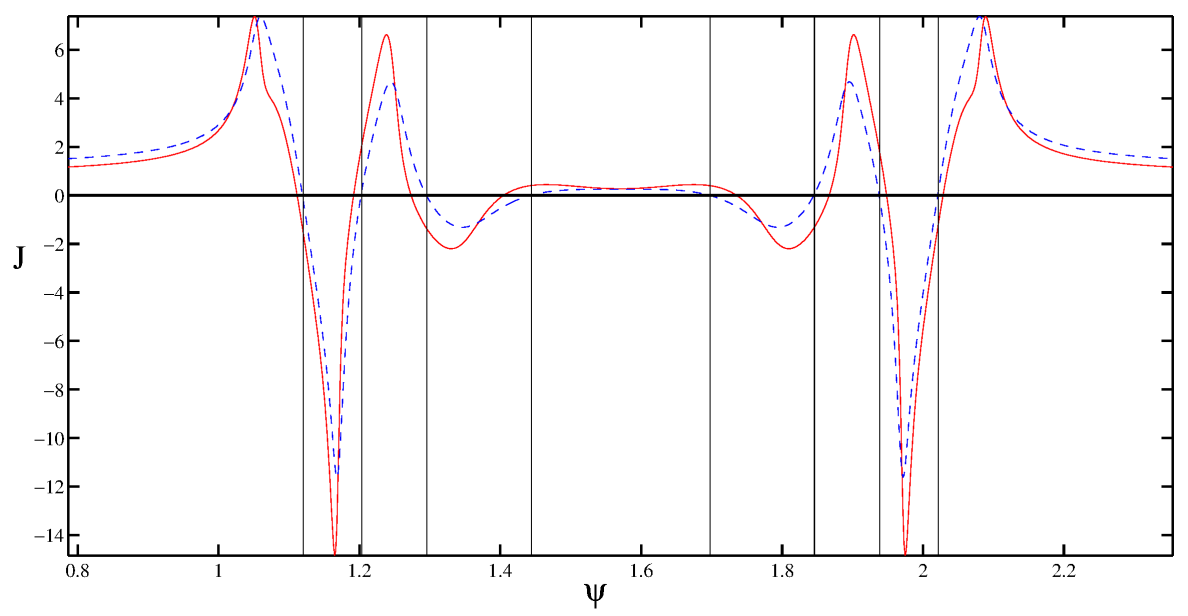

Figure 6. The Jacobians $J$ (dashed line) and $\widetilde{J}$ (solid line) at time $t=2000$ for the bifurcation parameter value $b_{2}=280$.

sample 3D plot of the computed asymptotic solution is shown in Fig. 7.

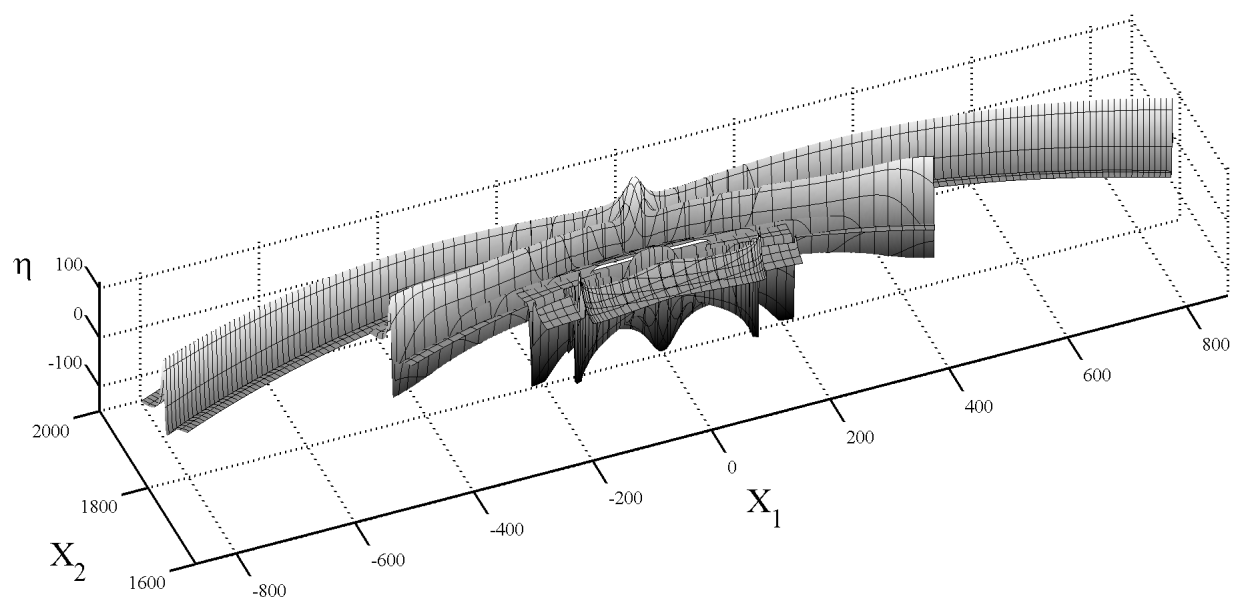

FiguRE 7 . The free water surface elevation at time $t=2000$ for the bifurcation parameter value $b_{2}=280$. 
We see that there is indeed a wave train moving along the elongated underwater bank and that the wave pattern in the train closely resembles the pattern of the cascade of caustics shown for the corresponding time in Fig. 4.

\section{Conclusion}

We use the problem on the propagation of waves generated by instantaneous localized sources over elongated underwater banks to show that, in the multidimensional case, such sources can generate wave trains even in dispersion-free (but inhomogeneous) media. This phenomenon is related to focusing effects and the bifurcation of cascades of nested space-time caustics.

Acknowledgements. The authors are grateful to Michael Berry for useful discussions. The research was supported by RFBR grants nos. 11-01-00973 and 12-01-31196.

\section{References}

[1] V. Arnold, A. Varchenko, S. Gussein-Zade. Singularities of differentiable maps. Nauka, Moscow, 1984. (Russian)

[2] V. M. Babich, V. S. Buldyrev. Asymptotic methods in short wave diffraction problems. Nauka, Moscow, 1972. (Russian)

[3] M. V. Berry, D. H. J. O'Dell. Ergodicity in wave-wave diffraction. J. Phys. A: Math. Gen., 32 (1999), $3571-3582$.

[4] M. V. Berry, C. Upstill. Catastrophe optics: morphologies of caustics and their diffraction patterns. Prog. Opt., 18 (1980), 257-346.

[5] S. Yu. Dobrokhotov. Asymptotic behavior of water surface waves trapped by shores and irregularities of the bottom relief. Dokl. Akad. Nauk SSSR, 289:3 (1986), 575-579. English transl., Soviet Phys. Dokl., 31:7 (1986), 537-539.

[6] S. Yu. Dobrokhotov, R. Nekrasov, B. Tirozzi. Asymptotic solutions of the linear shallow water equations with localized initial data. J. Engng Math., 69:2 (2011), 225-242.

[7] S. Dobrokhotov, M. Rouleux. The semi-classical Maupertuis-Jacobi correspondence for quasi-periodic Hamiltonian flows with applications to linear water waves theory. Asymptotic Analysis, 74:1-2 (2011), 33-73.

[8] S. Yu. Dobrokhotov, S. Ya. Sekerzh-Zenkovich. A class of exact algebraic localized solutions of the multidimensional wave equation. Mat. Zametki, 88:6 (2010), 942-945. English transl., Math. Notes, 88:6 (2010), 894-897.

[9] S. Yu. Dobrokhotov, S. Ya. Sekerzh-Zenkovich, B. Tirozzi, T. Ya. Tudorovskiy. Description of tsunami propagation based on the Maslov canonical operator. Dokl. Ross. Akad. Nauk, 409:2 (2006), 171-175. English transl., Russian Acad. Sci. Dokl. Math., 74:1 (2006), 592-596.

[10] S. Yu. Dobrokhotov, S. Ya Sekerzh-Zenkovich, B. Tirozzi, B. Volkov. Explicit asymptotics for tsunami waves in framework of the piston model. Russian J. Earth Sci., 8:ES403, 1-12 (2006).

[11] S. Yu. Dobrokhotov, S. Ya Sekerzh-Zenkovich, B. Tirozzi, B. Volkov. Asymptotic description of tsunami waves in the framework of the piston model: The general constructions and explicitly solvable models. Fund. Appl. Geophysics, 2 (2009), 15-29. (Russian)

[12] S. Dobrokhotov, A. Shafarevich, B. Tirozzi. Localized wave and vortical solutions to linear hyperbolic systems and their application to the linear shallow water equations. Russian J. Math. Phys., 15:2 (2008), 192-221.

[13] S. Yu. Dobrokhotov, B. Tirozzi, C. A. Vargas. Behavior near the focal points of asymptotic solutions to the Cauchy problem for the linearized shallow water equations with initial localized perturbations. Russian J. Math. Phys., 16:2 (2009), 228-245.

[14] S. Yu. Dobrokhotov, P. N. Zhevandrov. Nonstandard characteristics and Maslov's operator method in linear problems on transient water waves. Funktsional. Anal. i Prilozhen., 19:4 (1985), 43-54. English transl., Functional Anal. Appl., 19:4 (1985), 285-295.

[15] S. F. Dotsenko, B. Yu. Sergievskii, L. V. Cherkasov. Space tsunami waves generated by alternating displacement of the ocean surface. Tsunami Research, 1 (1986), 7-14. (Russian)

[16] J. J. Duistermaat. Oscillatory integrals, Lagrange immersions and unfolding of singularities. Comm. Pure Appl. Math., 27 (1974), 207-281.

[17] M. V. Fedoryuk. Mountain pass method. Moscow, Nauka, 1977.

[18] R. M. Garipov. Transient waves over an underwater ridge. Dokl. Akad. Nauk SSSR, 161:3 (1965), 547-550. (Russian)

[19] D. A. Indeytsev, N. G. Kuznetsov, O. V. Motygin, Yu. A. Mochalova. Trapped linear waves. St.-Petersburg State University Press, St.-Petersburg, 2007. (Russian)

[20] J. B. Keller. Surface waves on water of nonuniform depth. J. Fluid Mech., 4 (1958), 607-614.

[21] P. H. LeBlond, L. A. Mysak. Waves in the ocean. Elsevier, Amsterdam, 1978.

$[22]$ D. A. Lozhnikov. Analytic-numerical description of asymptotic solutions of a Cauchy problem in a neighbourhood of singularities for a linearized system of shallow water equations. Russian J. Math. Phys., 19:1 (2012), 44-62.

[23] V. S. Matveev. The Asymptotic eigenfunctions of the operator $\nabla D(x, y) \nabla$ corresponding to liouville metrics and waves on water captured by bottom irregularities. Mat. Zametki, 64:3 (1998), 414-422. English transl., Math. Notes, $64: 3$ (1998), 357-363. 
[24] C. C. Mei. The applied dynamics of ocean surface waves. World Scientific, Singapore, 1989.

[25] E. N. Pelinovskii. Hydrodynamics of tsunami waves. Nizhni Novgorod, 1996. (Russian)

[26] S. Ya. Sekerzh-Zenkovich. Simple asymptotic solution to the Cauchy-Poisson problem for leading waves. Russian J. Math. Phys., 16:2 (2009), 215-222.

[27] R. Thom. Structural stability and morphogenesis. Advanced Books Classics. Benjamin, Reading, 1975.

[28] B. R. Vainberg. Asymptotic methods in equations of mathematical physics. Moscow University, Moscow, 1982. English transl., Gordon and Breach, New York, 1989.

[29] S. Wang. The Propagation of the Leading Wave. ASCE Specialty Conference on Coastal Hydrodynamics, University of Delaware, June 29-July 1 (1987), 657-670. 